\title{
El tiempo de trabajo en plataformas: ausencia de jornada mínima, gamificación e inseguridad algorítmica
}

\section{Working time in platform work: absence of minimum working time, gamification and algorithmic insecurity}

\author{
Anna Ginès i Fabrellas* \\ Profesora Titular Derecho del Trabajo \\ Universitat Ramon Llull - Esade \\ ORCID ID: 0000-0001-6313-8355
}

Recibido: $14 / 12 / 2020$

Aceptado: 23/1/2021

doi: https://doi.org/10.20318/labos.2021.6045

Resumen: $\quad$ La finalidad del presente artículo es analizar la configuración y determinación
del tiempo de trabajo en el trabajo en plataformas. El trabajo en plataformas
está generando una clara precariedad laboral que va más allá de la naturaleza
jurídica de la relación de los trabajadores de plataformas. El singular modelo de
negocio de las plataformas digitales, basado en la contratación a demanda tiene
un claro impacto en la configuración del tiempo de trabajo en la economía de
plataformas que, como se analiza en el presente artículo, se traduce en empleo
precario dada la ausencia de un tiempo mínimo de trabajo garantizado y la
inseguridad algorítmica en la determinación del tiempo de trabajo.
Palabras clave:
trabajo en plataformas, tiempo de trabajo, jornada mínima garantizada, inse-
guridad algorítmica.

Abstract: The aim of this paper is to analyze the configuration and determination of the working time in platform work. Platforms work is clearly contributing to job insecurity beyond the classification of platform workers. The unique business model of digital platforms, based on on-demand hiring, has a clear impact on the determination of working time in the platform economy which, as analyzed in this article, translates into precarious employment given the absence of a guaranteed minimum working time and algorithmic insecurity in determining working time.

Keywords: $\quad$ platform work, working time, guaranteed minimum working time, algorithmic insecurity.

El presente artículo se ha realizado en el contexto del proyecto LABORAlgorithm (Algoritmos y relación laboral. Tratamiento jurídico del uso de Big Data, tecnología inteligente y algoritmos para la elaboración de perfiles y toma de decisiones automatizada en el ámbito laboral), financiado por FEDER/Ministerio de Ciencia, Innovación y Universidades - Agencia Estatal de Investigación/ _ Proyecto PGC2018-100918-A-100.

El presente estudio se ha cerrado a fecha de 19 de marzo de 2021. 


\section{Introducción}

"Antes de Internet, hubiese sido muy dificil encontrar a alguien, sentarlo durante diez minutos y hacer que trabajara para ti, y luego despedirlo al finalizar esos diez minutos. Pero con la tecnología, puede encontrarlos, pagarles una pequeña cantidad de dinero y luego deshacerte de ellos cuando ya no los necesites"1.

Con esta cita, Lukas Biewald, fundador de la plataforma Crowdflower, actualmente denominada FigureEight, define de forma desacomplejadamente precisa el modelo de trabajo en plataformas digitales, que utiliza personas como un objeto o una fuente inagotable de trabajo, como si se tratara de abrir el grifo, como se ilustra en una portada de diciembre de 2014 "Workers on tap" de The Economist ${ }^{2}$.

Resulta interesante, no obstante, esta cita, por cuanto se observa en ella la importancia de la tecnología como eje vertebrador del modelo de negocio desarrollado por las plataformas digitales; la tecnología resulta esencial para garantizar el modelo de contratación a demanda y externalización infinita que desarrollan las plataformas digitales. Además, esta cita resulta interesante por cuanto ilustra como se configura el tiempo de trabajo en plataformas digitales, esencialmente como micro jornadas de escasa o, incluso, ridícula duración con personas contratadas minuto a minuto.

El conflicto jurídico y social más importante en relación con el trabajo en plataformas digitales es su naturaleza jurídica; es decir, la consideración de las personas proveedoras del servicio como trabajadores por cuenta propia o por cuenta ajena.

En este sentido, existen pronunciamientos a favor y en contra del carácter laboral del trabajo en plataformas en prácticamente todos los países que han analizado la cuestión ${ }^{3}$. En el estado español, como se analiza en las páginas siguientes, existen también sentencias contradictorias en esta materia, si bien recientemente se ha pronunciado el Pleno de la Sala Social del Tribunal Supremo a favor de la existencia de relación laboral ${ }^{4}$. Esta sentencia, si bien clara y contundente, no parece resolver el conflicto de la naturaleza jurídica del trabajo en plataformas, por cuanto algunas plataformas de reparto de comida declararon su intención de mantener su modelo de negocio en tanto no exista un cambio legislativo5.

Más allá, el actual debate acerca del trabajo en plataformas parece limitarse a las plataformas de reparto o de transporte, tales como Glovo, Deliveroo, Uber Eats, etc., dejando de lado las plataformas de (micro)tareas online o de o crowdemployment ${ }^{6}$, como Amazon Mechanical Turk, Figure

\footnotetext{
${ }^{1}$ Moshe Z. Marvit, "How crowdworkers became the ghosts in the digital machine", The Nation, 5.2.2014 (disponible en: https://www.thenation.com/article/how-crowdworkers-became-ghosts-digital-machine/; última consulta: 27.11.2020).

2 "Workers on tap. The on-demand economy", The Economist, 30.12.2014 (disponible en: https://www.economist.com/leaders/2014/12/30/workers-on-tap; última consulta: 27.11.2020).

${ }^{3}$ Véase Ignasi Beltrán de Heredia Ruiz, "Employment status of platform workers (national courts decisions overview Australia, Brazil, Chile, France, Italy, United Kingdom, United States \& Spain), Blog Una mirada critica a las relaciones laborales, (disponible en: https://ignasibeltran.com/2018/12/09/employment-status-of-platform-workers-national-courts-decisions-overview-australia-brazil-chile-france-italy-united-kingdom-united-states-spain; última consulta: 27.11.2020).

${ }^{4}$ STS, 4a, 25.9.2020 (recurso núm. 4746/2019).

${ }^{5}$ Ver Carlos R. Cózar, "Glovo desafía al Tribunal Supremo: "Vamos a seguir trabajando con el mismo modelo", EDeconomíaDigital, 27.11.2020 (disponible en: https://www.economiadigital.es/finanzas-y-macro/glovo-desafia-al-tribunal-supremo-vamos-a-seguir-trabajando-con-el-mismo-modelo_20108986_102.html; última consulta: 27.11.2020).

${ }^{6}$ Valerio de Stefano y Antonio Aloisi, "Fundamental labour rights, platform work and human-rights protection of nonstandard workers", en Janice R. Bellace y Beryl ter HAAR (Editores), Research Handbook on Labour, Business and Human Rights Law, Edward Elgar, Reino Unido, 2019, p. 363.
} 
Eight o Upwork, dedicadas a la realización de tareas como traducción, transcripción de textos, descripción de imágenes, etc.

En esta línea, en la mesa de diálogo entre los agentes sociales para la regulación del trabajo en plataformas digitales ${ }^{7}$ una de las divergencias más importantes ha sido el alcance que debe tener la denominada "Ley Rider", debiéndose limitar solamente a las plataformas de reparto o mensajería o extendiéndose también a las plataformas de (micro)tareas online ${ }^{8}$. Y la propuesta final de regulación que ha propuesto el Gobierno, si bien incluye una presunción de laboralidad del trabajo en plataformas, se limita a las plataformas de reparto. ${ }^{9}$

En el contexto del debate social y político sobre la regulación del fenómeno del trabajo en plataformas, el objeto del presente artículo es analizar la configuración y determinación del tiempo de trabajo en el contexto de esta nueva forma de organización del trabajo. El trabajo en plataformas está generando una clara precariedad laboral que va más allá de la naturaleza jurídica de la relación de los trabajadores de plataformas. El singular modelo de negocio de las plataformas digitales, basado en la contratación a demanda, tiene un claro impacto en la configuración del tiempo de trabajo en la economía de plataformas que, como se analiza en el presente artículo, se traduce en empleo precario dada la ausencia de un tiempo de trabajo mínimo garantizado, estrategias de gamificación e inseguridad algorítmica. En este sentido, el artículo incluye una propuesta de regulación del tiempo de trabajo en el trabajo en plataformas como medida necesaria para avanzar en la protección social de esta nueva forma de organización del trabajo.

\section{La gig economy y la huida del Derecho del Trabajo}

Las plataformas digitales de la gig economy ${ }^{10}$-también denominada uber u on-demand economy ${ }^{11}$ han transformado la forma de organización del trabajo en la empresa gracias al uso intensivo de la tecnología y un singular modelo de negocio basado, como se analiza a continuación, en cuatro elementos esenciales ${ }^{12}$.

En primer lugar, la división del trabajo en microtareas. Las plataformas digitales han sustituido la configuración del servicio como una unidad por tareas individuales de muy corta duración,

\footnotetext{
${ }^{7}$ Ver la consulta pública previa a la elaboración de dicho proyecto normativo del 6.6.2020 (disponible en: http://www.mites.gob. es/ficheros/participacion/historico/consulta-publica/2020/Proyecto_08_20200606_consulta_publica_gabinete_empleo.pdf; última consulta: 27.11.2020).

${ }^{8}$ Ver "Gobierno y sindicatos acuerdan ampliar la ley de los "riders" a todas las plataformas digitales", Cinco Días, 21.10.2020 (disponible en: https://cincodias.elpais.com/cincodias/2020/10/21/economia/1603298049_863484.html; última consulta: 27.11.2020) y Gabriel Ubieto, “Trabajo limita el alcance de la nueva "Ley Rider" para atraer a la patronal”, El Periódico, 29.11.2020 (disponible en: https://www.elperiodico.com/es/economia/20201129/trabajo-limita-el-alcance-de-la-nueva-ley-rider-para-atraer-a-ceoe-8223215; última consulta: 29.11.2020).

${ }^{9}$ Ver Gabriel Ubieto, "Trabajo pacta con patronal y sindicatos que los riders sean, por defecto, asalariados", El Periódico, 10.2.2021 (disponible en: https://www.elperiodico.com/es/economia/20210210/trabajo-pacta-patronal-sindicatos-riders-11509236 [última consulta: 25.2 .2021$])$.

${ }^{10}$ Valerio DE STEFAno, "The rise of the "just-in-time workforce»: On-demand work, crowdwork and labour protection in the "gig-economy»", Conditions of Work and Employment Series, no 71, Organización Internacional del Trabajo, 2016.

${ }^{11}$ Steven Hill, "The future of work in the uber economy. Creating a safety net in a multi-employer world", Boston Review, 22.7.2015 (disponible en: http://bostonreview.net/us/steven-hill-uber-economy-individual-security-accounts; última consulta: 13.12.2020) o Mike JACONI, "The on-demand economy is revolutionizing consumer behaviour - Here's how", Business insider, 13.7.2014 (disponible en: https://www.businessinsider.com/the-on-demand-economy-2014-7?IR=T; última consulta: 13.12.2020).

${ }^{12}$ Para un análisis más en detalle, ver Anna Ginès I Fabrellas, El trabajo en plataformas digitales. Nuevas formas de precariedad, Thomson Reuters Aranzadi, Cizur Menor (Navarra), 2021.
} 
autónomas e independientes unas de las otras. A modo de ejemplo, en lugar de entender el servicio de reparto de comida a domicilio como una unidad, se divide en repartos individuales de tal manera que cada petición por parte de una persona clienta es un servicio autónomo e independiente.

En segundo lugar, el crowdsourcing ${ }^{13}$. Las plataformas digitales utilizan una nueva modalidad de externalización productiva, denominada crowdsourcing. Mediante una llamada u open call, la plataforma intenta atraer a un número elevado de personas dispuestas a prestar servicios en la plataforma. La finalidad es atraer al máximo número de personas para contar con oferta suficiente para cubrir la demanda que pueda existir en la plataforma en cualquier momento. El servicio, por tanto, es íntegramente externalizad a este elevado número de personas (de ahí el uso del término en inglés de crowd, que, como es bien sabido, significa multitud). Las plataformas digitales, por tanto, no disponen de la plantilla necesaria -al menos a priori- para la prestación del servicio, sino que es prestado de forma íntegramente descentralizada mediante personas externas, que son contratadas para la realización de las distintas tareas.

En tercer lugar, las plataformas digitales emplean la contratación a demanda u on-demand ${ }^{14}$. Cada una de las microtareas es externalizada a una persona proveedora del servicio en el momento en que se produce la demanda del servicio. Es decir, las personas que prestan servicios en el marco de plataformas digitales no son contratadas de forma estable, sino que son contratadas en el momento en que se origina la demanda del servicio y por el tiempo que dura su prestación. Esta contratación a demanda o por gigs da el nombre al fenómeno de la gig economy o economía de bolos.

La tecnología, como se ha apuntado, juega un papel esencial en la transformación de la forma de organización del trabajo en plataformas, por cuanto es la que permite la contratación a deman$\mathrm{da}^{15}$, pieza central del singular modelo de negocio de las plataformas digitales. Efectivamente, la tecnología de última generación permite identificar el momento exacto y preciso que una persona solicita el servicio y, en cuestión de segundos, esta petición es asignada a una persona dispuesta a llevarlo a cabo. Mediante el uso de sofisticados algoritmos, las plataformas digitales pueden intermediar en la asignación entre demanda y oferta del servicio en cuestión de segundos y sin incurrir en grandes costes de gestión.

Finalmente, en cuarto lugar, el modelo de negocio de las plataformas digitales se fundamenta en la contratación de personas que tienen la consideración formal de trabajadoras autónomas. En lugar de contratar a personas internamente para la prestación de servicios, las plataformas digitales ofrecen el servicio de forma íntegramente externalizada mediante personas que trabajan por cuenta propia y son contratadas servicio a servicio. Estas personas tienen la consideración formal de trabajadoras autónomas por cuanto aportan medios propios para la prestación de servicios y asumen los costes asociados a la actividad.

La combinación de estos cuatro elementos característicos del modelo de negocio de las plataformas digitales genera una novedosa transformación en la organización del trabajo, que permite la sustitución de formas más o menos estables de trabajo por cuenta ajena por formas de trabajo autónomo de muy corta duración.

Sin perjuicio de las ventajas que, desde un punto de vista empresarial, pueda generar este modelo de negocio -esencialmente, la reducción de costes de transacción ${ }^{16}$ y laborales ${ }^{17}-$, procede

\footnotetext{
${ }^{13}$ Ver Jeff Howe, “The rise of crowdsourcing”, Wired, 1.6.2006 (disponible en: http://www.wired.com/2006/06/crowds/; última consulta: 27.11 .2020$)$.

14 "Workers on tap. The on-demand economy", The Economist, op. cit.

${ }^{15}$ Daren C. Bradham, Crowdsourcing, MIT Press, Cambridge, 2013, p. 11-12.

${ }^{16}$ Brishen Rogers, "The Social Costs of Uber", The University of Chicago Law Review Dialogue, vol. 82, 2015, p. 86-89; Manuel Alejandro Hidalgo, El empleo del futuro. Un análisis del impacto de las nuevas tecnologías en el mercado laboral, Deusto, Barcelona, 2018, p. 4, entre otras referencias.

${ }^{17}$ Véase Birgitta Bergvall-Kåreborn y Debra Howcroft, "Amazon Mechanical Turk and the commodification of labor",
} 
advertir de los riesgos sociales y, en concreto, de la huida del Derecho del Trabajo ${ }^{18}$ que diseña el ecosistema propio de las plataformas digitales. Efectivamente, se trata de un modelo de negocio basado en el ahorro de costes laborales ${ }^{19}$, no solamente por la consideración -al menos, formalmente- del trabajo como trabajo autónomo, sino por diseñar una organización del trabajo que traslada costes y riesgos propiamente empresariales a las personas trabajadoras ${ }^{20}$.

\section{El trabajo en plataformas digitales: ¿̨confusión entre trabajo autónomo y trabajo por cuenta ajena?}

El conflicto jurídico y social más importante en relación con el trabajo en plataformas digitales es su naturaleza jurídica; es decir, la consideración de las personas proveedoras del servicio como trabajadores por cuenta propio o por cuenta ajena.

Como se ha apuntado anteriormente, existen pronunciamientos a favor y en contra del carácter laboral del trabajo en plataformas en prácticamente todos los países que han analizado la cuestión ${ }^{21}$. El ordenamiento jurídico español no es la excepción, con múltiples sentencias a favor del carácter autónomo del trabajo en plataformas ${ }^{22}$ y múltiples más a favor de su laboralidad ${ }^{23}$. Si bien recientemente se ha pronunciado el Pleno de la Sala Social del Tribunal Supremo a favor de la existencia de relación laboral ${ }^{24}$, no parece una cuestión resuelta, por cuanto algunas plataformas de reparto de comida ya han declarado su intención de mantener su modelo de negocio en tanto no exista un cambio legislativo ${ }^{25}$. Más allá, en la mesa de diálogo con los agentes sociales para la regulación del trabajo en plataformas digitales no ha existido consenso en cuanto a la forma de abordar el fenómeno ni tampoco el tratamiento jurídico que corresponde y la última propuesta de "Ley Rider" del Gobierno, que establece una presunción del trabajo en plataformas de reparto, no parece contentar a nadie.

El singular modelo de negocio de las plataformas digitales ha introducido novedades en cuanto a la organización del trabajo que cuestionan el encaje de esta forma de trabajo en el concepto de trabajo por cuenta ajena. Efectivamente, el trabajo en plataformas comparte elementos tradicionalmente propios del trabajo autónomo que parecen, a priori, alejarlo del trabajo por cuenta ajena. La tecnología de última generación, como se analiza a continuación, ha introducido nuevos elementos de flexibilidad que parecen asimilar el trabajo en plataformas a trabajo autónomo.

New Technology, Work and Employment, vol. 29, no 3, 2014, p. 215 o Jeremias Prassl y Martin Risak, "Uber, TaskRabbit, \& Co: Platforms as Employers? Rethinking the Legal Analysis of Crowdwork", Comparative Labor Law Policy Journal, vol. 37, no 3, 2016, p. 7, entre otras referencias.

${ }^{18}$ Véase Emanuele Dagnino, "Uber law: perspectiva jurídico-laboral de la sharing / on-demand economy”, Revista Internacional y Comparada de Relaciones Laborales y Derecho del Empleo, vol. 3, n. 3, 2015, p. 15-25.

${ }^{19}$ Como afirma Jeremias Adams-Prassl, la elusión de la norma laboral se encuentra en el centro del modelo de negocio de las plataformas digitales (Jeremias Prassl, Humans as a service. The promise and perils of work in the gig economy, Oxford University Press, Nueva York, 2018, p. 21).

${ }^{20}$ Brishen Rogers, "The Social Costs of Uber", op. cit., p. 98; o Sangeet Paul Choudary, "The architecture of digital labour platforms: policy recommendations on platform design for worker well-being", ILO Future of Work Research Paper Series, no 3, 2018 , p. 13.

${ }^{21}$ Véase Ignasi Beltrán de Heredia Ruiz, "Employment status of platform workers...”, op. cit.

${ }^{22}$ Entre las más destacadas se encuentra la STSJ Madrid de 19.9.2019 (recurso núm. 195/2019), si bien el tribunal modifica su posición en las STSJ Madrid de 27.11.2019 (recurso núm. 588/2019), 18.12.2019 (recurso núm. 714/2019), 17.1.2020 (recurso núm. 1323/2019) y 3.2.2020 (recurso núm. 749/2019) y la sentencia ha sido casada y anulada por la STS, 4a , 25.9.2020 (recurso núm 4746/2019).

${ }^{23}$ STS, 4a, 25.9.2020 (recurso núm 4746/2019).

${ }^{24}$ Ver, entre las más relevates, STSJ Asturias de 25.7.2019 (rec. no 1143/2019), Madrid de 27.11.2019 (recurso núm. 588/2019), 18.12.2019 (recurso núm. 714/2019), 17.1.2020 (recurso núm. 1323/2019), 3.2.2020 (recurso núm. 749/2019), Catilla y León de 17.2.2020 (recurso núm. 2253/2019) y Cataluña de 21.2.2020 (recurso núm. 5613/2019), 7.5.2020 (recurso núm. 5647/2019), 12.5.2020 (recurso núm. 6774/2019), 16.6.2020 (recurso núm. 5997/2019).

${ }^{25}$ Ver Carlos R. Cózar, "Glovo desafía al Tribunal Supremo: "Vamos a seguir trabajando con el mismo modelo”, EDeconomíaDigital, 27.11.2020 (disponible en: https://www.economiadigital.es/finanzas-y-macro/glovo-desafia-al-tribunal-supremo-vamos-a-seguir-trabajando-con-el-mismo-modelo_20108986_102.html; última consulta: 27.11.2020). 
Asimismo, las propias plataformas han contribuido a la confusión creando una nueva y sofisticada terminología ${ }^{26}$, diseñada para eludir la norma laboral ${ }^{27}$, que genera la idea de estar ante un nuevo y novedoso fenómeno laboral que no encuentra cabida en las viejas y arcaicas estructuras laborales.

\subsection{Argumentos a favor del trabajo autónomo: medios de producción, costes, capacidad para determinar el tiempo de trabajo y para aceptar y rechazar encargos}

En primer lugar, como se ha apuntado anteriormente, las personas que trabajan en plataformas utilizan medios de producción propios (bicicleta, motocicleta, coche, ordenador, móvil, etc.) y asumen los costes asociados a la prestación de servicios (gasolina, conexión a Internet, etc.). Se trata de medios y costes esenciales para la prestación de servicios, que son propiedad y asumidos por las personas trabajadoras, sin que sean puestos a disposición por parte de la plataforma -si bien en ocasiones, la plataforma los proporciona a cambio de un precio o fianza ${ }^{28}$ - Según establece la sentencia del Tribunal Superior de Justicia de Madrid de 19.9.2019 ${ }^{29}$, que se pronunció en contra de la laboralidad del trabajo en la plataforma Glovo, la utilización de medios propios y la asunción de los costes de la actividad excluye la existencia de ajenidad.

En segundo lugar, como declara el Tribunal Superior de Justicia de Madrid en la sentencia anteriormente citada, la asunción del riesgo y ventura también se observa en la responsabilidad de las personas que reparten para la plataforma Glovo de responder por el buen fin del servicio, por cuanto asumen, frente a la persona cliente final, los dańos o pérdidas que pueda padecer el producto o mercancía transportada. Incluso, algunas plataformas - como es el caso de Amazon Mechanical Turkimponen cláusulas de satisfacción en los contratos, en virtud de las cuales la persona trabajadora no recibe el precio acordado en caso de que la empresa clienta no quede satisfecha con los resultados ${ }^{30}$.

En tercer lugar, las personas que trabajan en plataformas mayoritariamente reciben una retribución directamente proporcional al número de servicios realizados, indicio propio del trabajo autónomo ${ }^{31}$. También este elemente ha sido entendido como excluyente de la ajenidad propia de la relación laboral, por cuanto implica la asunción del riesgo y ventura de la actividad propio del trabajo autónomo ${ }^{32}$. Según la sentencia del Juzgado de lo Social no 1 de Salamanca de 14.6.201933, no existe ajenidad en la prestación de servicios, en tanto que la persona trabajadora "percibía sus retribuciones, no de acuerdo con una cuota fija ni determinada cada mes, sino que variaban en función de los encargos o entregas realizadas, a la que se añadia una parte variable en función de los kilómetros realizados y el tiempo de espera".

En cuarto lugar, y probablemente uno de los argumentos más importantes a efectos de descartar la existencia de relación laboral en el trabajo en plataformas, las personas que trabajan en plataformas tienen capacidad para determinar su tiempo de trabajo; capacidad para determinar, no

\footnotetext{
${ }^{26}$ Sarah KessLer, Gigged. The Gig Economy, the end of the job and the future of work, Business Books, Londres, 2018, p. 97.

${ }^{27}$ Sarah BUTLER, "Deliveroo accused of "creating vocabulary" to avoid calling couriers employees", The Guardian, 5.4.2017 (disponible en: https:/www.theguardian.com/business/2017/apr/05/deliveroo-couriers-employees-managers; última consulta: 27.11.2020).

En sentido similar, ver hecho probado decimocuarto SJS no 31 de Barcelona de 11.6.2019 (no 193/2019).

${ }^{28}$ SJS no 1 Gijón de 20.2.2019 (sentencia nº 61/2019).

${ }^{29}$ Recurso núm. 195/2019.

${ }^{30}$ https://docs.aws.amazon.com/AWSMechTurk/latest/AWSMechanicalTurkGettingStartedGuide/SvcIntro.html.

${ }^{31}$ Ver Maria Cesira Urzí Brancati, Annarosa Pesole y Enrique Fernández-Macías, New evidence on platform workers in Europe. Results from the second COLLEEM survey, JRC Science for Policy Report, Publications Office of the European Union, Luxemburgo, 2020, p. 36.

${ }^{32}$ Ver STSJ Madrid de 19.9.2019 (recurso núm. 195/2019).

${ }^{33}$ Sentencia núm. 215/2019
} 
solamente el horario -elemento compatible, como establece el Tribunal Supremo con el trabajo por cuenta ajena ${ }^{34}-$, sino su tiempo de conexión a la plataforma; es decir, capacidad para determinar cuántas horas al día, a la semana o al mes se conectan e, incluso, capacidad para determinar si se conectan o no a la plataforma ${ }^{35}$.

En quinto lugar, otro elemento importante a efectos de determinar el carácter autónomo del trabajo en plataformas ha sido la capacidad de las personas que prestan servicios para seleccionar y rechazar encargos. Muchas plataformas digitales organizan el trabajo de tal manera que reconocen a las personas trabajadoras la capacidad para seleccionar los servicios a realizar en atención a sus intereses y la capacidad para rechazar servicios, incluso cuando han sido previamente aceptos. La capacidad para aceptar pedidos sin obligación de realizar un número mínimo y la capacidad de rechazar encargos es un elemento destacado para descartar la laboralidad del trabajo en plataformas, por cuanto evidencia autonomía organizativa y falta de dependencia al círculo rector de la empresa ${ }^{36}$.

Estos dos elementos añaden complejidad al debate acerca de la naturaleza jurídica del trabajo en plataformas, por cuanto se trata de dos elementos, ciertamente, propios y característicos del trabajo autónomo. La tecnología de última generación ha introducido elementos de flexibilidad hasta ahora desconocidos, que cuestionan -al menos cuando analizados de forma individual- el encaje de esta nueva organización del trabajo al concepto de trabajo por cuenta ajena.

Efectivamente, estos dos elementos han sido identificados como incompatibles con la relación laboral. Como establece la ya citada sentencia del Tribunal Superior de Justicia de Madrid de 19.9.2019 ${ }^{37}$, "[e] l trabajador decidia el momento de inicio y finalización de su jornada, asi como la actividad que realizaba durante la misma, seleccionando los pedidos que quería realizar y rechazando los que no quería. No tenía obligación de realizar un determinado número de pedidos, ni de estar activo un número de horas al día o a la semana ni tampoco la empresa indicaba los pedidos a realizar ni cuando debia empezar o finalizar su jornada". Similarmente entre la doctrina, se ha afirmado que la capacidad para determinar el tiempo y, más importantemente, el volumen de trabajo o la capacidad para rechazar encargos elimina necesariamente la existencia de dependencia respecto de la plataforma ${ }^{38}$.

Finalmente, otros elementos indicativos de trabajo autónomo identificados por las resoluciones judiciales ${ }^{39}$ que se han pronunciado en este sentido son la libertad para seleccionar la ruta, itinerario, procedimiento o medio de transporte para la ejecución de la actividad; la ausencia de necesidad de justificar ausencias; la ausencia de pacto de exclusividad y la prestación de servicios para terceros; o, incluso, la declaración de la persona trabajadora de prestar servicios en régimen de trabajo autónomo económicamente dependiente.

\subsection{Argumentos a favor del trabajo por cuenta ajena: dirección algoritmica, tecnología como infraes- tructura productiva esencial y falta de capacidad de emprendedoría}

Un análisis en profundidad de las características del trabajo en plataformas evidencia que, sin perjuicio de los anteriores elementos de flexibilidad introducidos por esta nueva modalidad de orga-

\footnotetext{
${ }^{34}$ Ver, entre las más recientes, STS, 4a, 6.10 .2010 (RJ 2010/5017) y 19.2.2014 (RJ 2014/2075).

${ }^{35}$ Ver STSJ Madrid de 19.9.2019 (recurso núm. 195/2019) y SJS no 39 de Madrid de 3.9.2018 (sentencia núm. 284/2018).

${ }^{36}$ Destacan, en este sentido, STSJ Madrid de 19.9.2019 (recurso núm. 195/2019), Cantabria 26.4.2019 (rec. no 237/2019) y SJS no 1 de Salamanca de 14.6.2019 (sentencia núm. 215/2019).

${ }^{37}$ Recurso núm. 195/2019.

${ }^{38}$ Ver, en este sentido, Jesús R. Mercader UGuina, El futuro del trabajo en la era de la digitalización y la robótica, Tirant lo Blanch, Valencia, 2017, p. 96 y Rubén Agote, "On demand economy: 10 claves de entendimiento laboral”, IUSLabor, no 1, 2017 , p. 14.

${ }^{39}$ STSJ Madrid de 19.9.2019 (recurso núm. 195/2019), SJS no 1 de Salamanca de 14.6.2019 (sentencia núm. 215/2019) o nº 17 de Madrid de 11.1.2019 (sentencia núm. 12/2019).
} 
nización del trabajo, existen indicios de laboralidad. Efectivamente, existen elementos propios de la organización del trabajo en plataformas que lo asimilan, indudablemente, al trabajo por cuenta ajena, al concurrir las notas de ajenidad y dependencia.

En primer lugar, las plataformas utilizan formas de dirección y control algorítmico ${ }^{40}$. Como se ha apuntado anteriormente, el modelo de organización del trabajo de las plataformas digitales confiere a las personas trabajadoras capacidad para determinar su tiempo y volumen de trabajo. Las personas que trabajan en plataformas tienen la capacidad para determinar el número de horas de conexión a la plataforma e, incluso, si quieren o no conectarse. Además, muchas plataformas reconocen también la capacidad de las personas trabajadoras de seleccionar los servicios a realizar e, incluso, rechazar servicios asignados. Si bien, ciertamente, estas son prácticas utilizadas en muchas plataformas, lo cierto es que la forma de dirección y organización algorítmica utilizada por las plataformas digitales condiciona significativamente esta capacidad, convirtiéndose en una verdadera forma de dependencia ${ }^{41}$-identificada, incluso, como "hiperdependencia"42

Las plataformas utilizan distintas estrategias para modular el comportamiento de las personas trabajadoras, en cuanto a tiempo de trabajo y aceptación de servicios, para encajar con las necesidades e intereses de la plataforma. Así, algunas plataformas reconocen incentivos económicos o aplican sistemas de discriminación de precios o algorithmic pricing ${ }^{43}$ para retribuir más a las personas que más se conectan a la plataforma o a las personas que se conectan en aquellos días u horas de más demanda. Otras plataformas utilizan un sistema de asignación de tareas o franjas horarias que beneficia a las personas con más horas de conexión, una elevada tasa de aceptación de servicios y una baja tasa de rechazos. Incluso, algunas plataformas ranquean a las personas trabajadoras estableciendo una prelación entre ellas en base a sus puntuaciones individuales y, posteriormente, distribuyen la asignación de tareas en atención a estas puntuaciones, beneficiando a aquellas con más tiempo de conexión y disponibilidad en la plataforma.

Los sistemas de geolocalización y control digital que utilizan las plataformas digitales permiten extraer una cantidad muy elevada de datos referentes a la actividad de las personas trabajadoras en la plataforma ${ }^{44}$. Así, la plataforma tiene información acerca de la geolocalización de la persona cuando está conectada a la plataforma, del número de horas de conexión, de la disponibilidad de la persona en días y horas de alta demanda, del número de pedidos aceptados, del número de pedidos rechazados, el tiempo de ejecución de cada servicio, satisfacción por parte de las personas o empresas clientas, etc. Cada servicio individual realizado por cada persona trabajadora es registrado, monitorizado y evaluado, colocando a las personas trabajadoras en una situación de control permanente ${ }^{45}$.

Los datos extraídos sobre la actividad de las personas en la plataforma son procesados e integrados en sofisticados algoritmos que toman decisiones de forma automatizada sobre asignación de

\footnotetext{
${ }^{40}$ Min Kyung Lee, Daniel Kusbit, Evan Metsky y Laura Dabbish, "Working with machines: the impact of algorithmic and data-driven management on human workers", Conference CHI'15 Proceedings of the 33rd Annual ACM Conference on Human Factors in Computing Systems, 2015, p. 1-10.

${ }^{41}$ Miriam A. Cherry, "Beyond misclassification: the digital transformation of work", Comparative Labor Law \& Policy Journal, vol. 37, no 3, 2016, p. 602; Alex Rosenblat y Luke STARK, "Algorithmic labor and information asymmetries: a case study of Uber's drivers", International Journal of Communication, vol. 10, 2016, p. 3777; Jeremias PRAssL, Humans as a service, op. cit., p. 52; Alan BoGG, "Escaping Labour Law's Matrix: a review essay", Law Research Paper Series, no 5, University of Bristol, 2019, p. 8; entre otras referencias.

${ }^{42}$ Miguel Rodríguez-Piñero Royo, "La figura del trabajador de plataforma: las relaciones entre las plataformas digitales y los trabajadores que prestan sus servicios”, en Francisco Pérez de los Cовоs (Director), El trabajo en plataformas digitales. Análisis sobre su situación jurídica y regulación futura, Wolters Kluwer, Las Rozas (Madrid), 2018 (versión digital).

${ }^{43}$ Alex Rosenblat y Luke Stark, "Algorithmic labor and information asymmetries...", op. cit., p. 3765-3766.

${ }^{44}$ Es interesante notar que el Tribunal Supremo, respecto de la plataforma Glovo, ha identificado el propio sistema de geolocalización también como "un indicio relevante de dependencia en la medida en que permite el control empresarial en tiempo real del desempeño de la prestación" (STS, 4a, 25.9.2020 (recurso no 4746/2019)).

${ }^{45}$ Henar Álvarez Cuesta, El futuro del trabajo vs. el trabajo del futuro. Implicaciones laborales de la industria 4.0, Colex, A Coruña, 2017, p. 112.
} 
tareas o preferencia para la elección de franjas horarias. Aquellas personas con mejores puntuaciones son beneficiadas con más tareas y preferencias en la elección de trabajo lo que, a su vez, repercute en más tareas y preferencia en la elección de trabajo.

Estas formas de dirección y organización algorítmica toman en consideración la actividad pasada de las personas en la plataforma para determinar su actividad futura; es decir, la actividad pasada de las personas determina su capacidad para generar ingresos en el futuro. En consecuencia, se convierten en instrumentos muy efectivos para modular el comportamiento de las personas e incentivar que se ajusten a los parámetros valorados por la plataforma. Como establece el Tribunal Supremo en su sentencia de 25.9.2020 respecto de la plataforma Glovo, la libertad de las personas trabajadoras para determinar su tiempo y volumen de trabajo está claramente condicionada por estas estrategias de la plataforma, por cuanto si la persona trabajadora "no está disponible para prestar servicios en las franjas horarias con más demanda, su puntuación disminuye y con ella la posibilidad de que en el futuro se le encarguen más servicios y conseguir la rentabilidad económica que busca, lo que equivale a perder empleo y retribución".

Las fórmulas de gamificación ${ }^{46}$ y dirección algorítmica que emplean las plataformas resultan muy efectivas para dirigir y gestionar la actividad laboral en la plataforma, especialmente respecto de aquellas personas que dependen económicamente del trabajo desarrollado en ella. Así, las personas que quieran utilizar el trabajo en plataforma como vía para obtener ingresos -ya sean principales o complementarios- tienen fuertes incentivos a adaptar su comportamiento en términos de tiempo de trabajo y aceptación de pedidos a las métricas o variables utilizadas por la plataforma y, así, acceder a trabajo en el futuro.

En segundo lugar, la existencia de relación laboral en el trabajo en plataformas se deriva también de la ausencia de ajenidad; las personas que trabajan en plataformas digitales no asumen el riesgo y ventura de la actividad.

Por un lado, es cierto que las personas que trabajan en plataformas digitales aportan medios de producción propios, tales como el móvil, ordenador, bicicleta, coche, etc. y asumen los gastos asociados a su actividad. Sin embargo, no es posible identificar la asunción del riesgo y ventura de la actividad, por cuanto estos elementos no son la infraestructura esencial y nuclear de la actividad. Los medios de producción esenciales y nucleares de la actividad prestada por las plataformas digitales son, precisamente, la propia plataforma, la aplicación informática y el algoritmo. Son estos los elementos económica y productivamente relevantes a efectos de valorar la existencia de ajenidad ${ }^{47}$. Efectivamente, desde un punto de vista económico, estos son los elementos que requieren mayor inversión económica y que, por tanto, implican la asunción de un riesgo de rentabilizar dicha inversión. También desde un punto de vista productivo son los elementos más relevantes, por cuanto son los que permiten la esencia de la organización del trabajo de las plataformas digitales: la rápida conexión entre oferta y demanda de un servicio. Según establece la STJUE 20.12.2017 ${ }^{48}$, sin dichos elementos los "conductores no estarian en condiciones de prestar servicios de transporte y, por tanto, las personas que desean realizar un desplazamiento urbano no podrían recurrir a los servicios de los mencionados conductores".

Por otro lado, la existencia de ajenidad también se evidencia en la falta de capacidad de emprendedoría de las personas trabajadoras. Es la plataforma la que incide en el mercado, tomando

\footnotetext{
${ }^{46}$ Sarah MAson, "High score, low pay: why the gig economy loves gamification", The Guardian, 20.11.2018 (disponible en: https://www.theguardian.com/business/2018/nov/20/high-score-low-pay-gamification-lyft-uber-drivers-ride-hailing-gig-economy?CMP=twt_gu; última consulta: 27.11.2020).

${ }^{47}$ Según establece el Tribunal Supremo, "la naturaleza laboral de la relación no se desvirtúa ni desaparece por el hecho de que el trabajador aporte su vehiculo propio, siempre que... tal aportación no tenga la relevancia económica necesaria para convertir su explotación en elemento fundamental de dicha relación, ni en la finalidad esencial del contrato, sino que, por el contrario, lo predominante es el trabajo personal del interesado, quedando configurado el vehiculo como una mera herramienta de trabajo" (STS, 4a , 5.11.1993 (RJ 1993/8547)).

${ }^{48}$ Asunto Asociación Profesional Élite Taxi v. Uber Systems Spain, S.L. (C-434/15).
} 
todas las decisiones económicas, estratégicas y comerciales relacionadas con el servicio y las condiciones del mismo -tales como precios, tarifas, mercados, acuerdos comerciales, zonas geográficas, marca ${ }^{49}$, etc.-, evidenciándose la existencia de ajenidad, como confirma la STS, 4a, 25.9.2020 ${ }^{50}$. Más allá, las plataformas digitales generalmente prohíben a las personas trabajadoras establecer contacto con personas usuarias fuera de la plataforma, limitando las oportunidades de negocio fuera del mercado interno de la plataforma. Las personas trabajadoras no tienen capacidad real alguna para adoptar decisiones que puedan influir en su supuesto negocio, más allá de incrementar el número de horas de conexión a la plataforma.

Por todo lo anterior, es posible concluir que el trabajo en plataformas es trabajo por cuenta ajena ex artículo 1.1 ET, por desarrollarse dentro del ámbito de dirección y organización de la plataforma, que adopta decisiones de distribución y organización del trabajo, y por existir ajenidad, al no asumir las personas trabajadoras el riesgo y ventura de la actividad.

\section{El tiempo de trabajo en plataformas digitales: nuevos elementos de precariedad}

El modelo de precariedad laboral desarrollado por las plataformas digitales va más allá de la incorrecta calificación del trabajo como trabajo autónomo. Efectivamente, como se analiza en el presente apartado, existen características intrínsecas de la organización del trabajo en plataformas digitales que contribuyen a generar una elevada precariedad laboral.

El trabajo en plataformas se configura como un trabajo esencialmente flexible ${ }^{51}$ y la flexibilidad que ofrece poder determinar el tiempo y volumen de trabajo es el principal reclamo utilizado por las plataformas digitales para atraer a personas dispuestas a prestar servicios en ella. "Sé tu propio jefe" o "Trabaja cuando quieras", publicitan algunas plataformas digitales ${ }^{52}$. Y, ciertamente, esta flexibilidad en materia de tiempo de trabajo es valorada positivamente por parte de las personas trabajadoras, por cuanto les permite acceder a fórmulas de trabajo que pueden ajustarse a la perfección a sus necesidades personales y familiares ${ }^{53}$. Sin embargo, sin perjuicio de los efectos positivos de la flexibilidad en materia de tiempo de trabajo, el presente apartado revela que viene con un precio de elevada precariedad.

En este sentido, en el contexto del debate social y político sobre la denominada "Ley Rider", es importante destacar que la declaración de laboralidad del trabajo en plataformas no resultará suficiente para garantizar la protección de esta nueva forma de trabajo. Existen elementos relacionados con el singular modelo de negocio de las plataformas digitales, esencialmente la contratación a demanda, que impactan negativamente en la calidad del trabajo ofertado en el marco de estas plataformas. La utilización de la contratación a demanda o just-in-time $e^{54}$ para la contratación de las

${ }^{49}$ En cuanto a la prestación bajo marca ajena como nuevo indicio de laboralidad, ver Adrián Todolí Signes, "Nuevos indicios de laboralidad como resultado de las nuevas empresas digitales", en Miguel C. Rodríguez-Piñero Royo y Macarena Hernández BEJARANO (Directores), Economia colaborativa y trabajo en plataforma: realidades y desafios, Editorial Bomarzo, Albacete, 2017, p. 237.

${ }^{50}$ Recurso no 4746/2019. Ver, en esta línea también, STSJ Asturias 25.7.2019 (recurso no 1143/2019).

${ }^{51}$ Según la encuesta COLLEEM II, la mayor parte de las personas que trabajan en plataformas digitales declaran disponer de flexibilidad en materia de tiempo de trabajo (Maria Cesira Urzí Brancati et al., New evidence on platform workers in Europe, op. cit., p. 45).

${ }^{52} \mathrm{Ver}$, a modo de ejemplo, https://www.uber.com/en-NL/blog/do-you-want-to-become-an-uber-partner-driver-with-uber-youare-your-own-boss/ o https:/glovoapp.com/es/glovers (última consulta: 13.12.2020).

${ }^{33}$ Ursula Huws, Neil H. Spencer, Dag S. Syrdal, Kaire Holts, Work in the European Gig Economy. Research results from the UK, Sweden, Germany, Austria, The Netherlands, Switzerland and Italy, Foundation for European Progressive Studies, UNI Europa y University of Hertfordshire, 2017, p. 40-41; Chris Forde, Mark Stuart, Simon Joyce, Liz Oliver, Danat Valizade, Gabriella Alberti, Kate Hardy, Vera Trappmann, Charles Umney y Calum Carson, The Social Protection of Workers in the Platform Economy, Directorate General for Internal Policies, Policy Department A: Economic and Scientific Policy, Parlamento Europeo, noviembre 2017, p. 51; o Annarosa Pesole, Maria Cesira Urzí Brancati, Enrique Fernández-Macías, Federico Biagi, F. e Ignacio González Vázquez, Platform Workers in Europe, JRC Science for Policy Report, Publications Office of the European Union, Luxemburgo, 2018, p. 43.

${ }^{54}$ Valerio de Stefano, "The rise of the «just-in-time workforce»...”, op. cit. 
personas trabajadoras impacta en el tiempo de trabajo, que se configura como altamente inestable. Esta configuración del tiempo de trabajo como intrínsecamente inestable se traduce, a mi entender, en una precariedad laboral extrema impropia de la regulación laboral. Según apunta un sector de la doctrina, las plataformas digitales están contribuyendo a la "uberización" del trabajo ${ }^{55}$ o la configuración del "e-precariado" 56 , que requiere mayor intervención que la declaración de laboralidad.

\subsection{Ausencia de jornada y retribución minimas garantizadas}

La consideración formal de las personas que trabajan en plataformas como trabajadoras por cuenta propia supone despojarlas de la protección propia de la relación laboral. En concreto a efectos del presente artículo, despojarlas de la protección laboral en materia de tiempo de trabajo: jornada laboral máxima, descansos mínimos, vacaciones retribuidas o medidas de conciliación de la vida laboral y familiar.

Las personas que trabajan en plataformas digitales realizan jornadas de trabajo superiores a las personas que trabajan en empresas de la economía tradicional. Esta es una de las conclusiones derivadas de la encuesta COLLEEM II ${ }^{57}$, como consecuencia también del hecho que el trabajo en plataformas digitales es mayoritariamente complementario a un trabajo en la economía tradicional. Adicionalmente, el trabajo en plataformas es mayoritariamente realizado fuera de horas típicas de trabajo o en horarios antisociales. Nuevamente según la encuesta COLLEEM II ${ }^{58}$, dos terceras partes de las personas que trabajan en plataformas digitales trabaja fines de semana y un porcentaje similar trabaja en horario nocturno.

Si bien formalmente libres para determinar su tiempo de trabajo y, por tanto, para determinar cuándo y cuanto trabajan, las personas que trabajan en plataformas digitales se encuentran fuertemente condicionadas a la hora de tomar dichas decisiones. El sistema de gestión y organización algorítmico utilizado por las plataformas, según el cual se asignan más tareas o de mejor valor a las personas más horas conectadas y/o en las horas de más demanda, induce a las personas trabajadoras a realizar largas jornadas laborales y en horarios antisociales para mantener su posición en la plataforma.

Más allá, y sin perjuicio de la importancia de la ausencia de protección frente a jornadas máximas y descansos mínimos, el trabajo en plataformas digitales plantea una forma de desprotección adicional: la ausencia de jornada mínima. La utilización de la contratación a demanda -por tanto, cuando existe demanda del mercado-, implica que las personas que trabajan en plataformas digitales no tienen una jornada mínima garantizada. La totalidad de su trabajo depende directamente de las circunstancias y exigencias del mercado y de la demanda existente en ese momento, sin que las plataformas, mayoritariamente, garanticen un tiempo de trabajo mínimo y, en consecuencia, una retribución mínima.

El trabajo en plataformas digitales y la utilización del esquema de contratación a demanda supone la utilización de facto del contrato de cero horas ${ }^{59}$. El contrato de cero horas, disponible en algunos ordenamientos jurídicos del entorno, implica que las personas son contratadas sin estar

\footnotetext{
${ }^{55}$ Ver, entre otras referencias, Philippe Auvergnon, "Angustias de uberización y retos que plantea el trabajo digital al Derecho Laboral", Revista Derecho Social y Empresa (número especial: Nuevas formas de trabajo. Retos presentes y futuros), n 6, 2016, p. 25-42; o Juana María Serrano García, "La "uberización" del trabajo: una forma más de violencia laboral”, en Lourdes Mella Méndez (Directora), Los actuales cambios sociales y laborales: nuevos retos para el mundo del trabajo, vol. 1, Peter Lang, Bern, 2017, p. 209-228.

${ }^{56}$ Henar Álvarez Cuesta, El futuro del trabajo vs. el trabajo del futuro, op. cit., p. 104.

${ }^{57}$ Maria Cesira Urzí Brancati et al., New evidence on platform workers in Europe, op. cit., p. 42.

${ }^{58}$ Maria Cesira Urzí Brancati et al., New evidence on platform workers in Europe, op. cit., p. 44.

${ }^{99}$ Para un análisis en detalle, ver Anna Ginès i Fabrellas, "The zero-hour contract in platform work. Should we ban it or embrace it?”, IDP. Revista de Internet, Derecho y Politica, no 28, 2019, p. 1-15.
} 
sujetas a un tiempo de trabajo mínimo, sino que solamente prestan servicios cuando son llamadas por la empresa. En sentido similar, el trabajo en plataformas digitales implica que las personas trabajadoras son "llamadas" a trabajar ante la petición de servicios por parte de personas clientas, sin que exista un tiempo mínimo de trabajo garantizado y, en consecuencia, tampoco una retribución mínima garantizada.

El contrato de cero horas, como se ha apuntado, es legal en ordenamientos jurídicos del entorno, como es el caso del Reino Unido ${ }^{60}$, Grecia ${ }^{61}$ o Italia ${ }^{62}$. Sin embargo, resulta ilegal en el ordenamiento jurídico-laboral espańol, al requerir la determinación de la jornada ordinaria (artículos 8.5 y 34.1 ET) y al existir límites a la realización de horas por encima de dicha jornada ordinaria (artículos 12.5 y 35 ET).

El contrato de cero horas genera una inestabilidad y precariedad laboral extrema ${ }^{63}$, al desplazar una parte importante del riesgo propiamente empresarial a las personas trabajadoras. Como consecuencia de la contratación a demanda y la dependencia absoluta de las adversidades del mercado, las personas trabajadoras asumen el riesgo de ausencia de demanda.

En este punto, resulta esencial destacar que en el trabajo en plataformas solamente se considera tiempo de trabajo -y, por tanto, tiempo retribuido- el tiempo que la persona trabajadora se encuentra ejecutando un servicio. Es decir, solamente es tiempo de trabajo el tiempo efectivo de prestación de servicios. En sentido contrario, el tiempo que la persona trabajadora se encuentra conectada a la plataforma y a la espera de recibir servicios o buscando tareas a realizar no es tiempo de trabajo y, por tanto, es tiempo no retribuido.

Esta constituye una diferencia importante respecto el contrato de cero horas tradicional. En el contrato de cero horas la persona trabajadora se encuentra o bien prestando servicios o bien absolutamente desvinculada de la empresa, sin que exista, como sucede en el trabajo en plataformas, un tiempo de disponibilidad; es decir, un tiempo que la persona se encuentra conectada e inmediatamente disponible para atender a peticiones de trabajo de la empresa.

En el trabajo en plataformas existe un porcentaje elevado del tiempo que la persona se encuentra conectada a la plataforma y disponible para recibir servicios, pero sin percibir remuneración por ello. Efectivamente, según un estudio de la Organización Internacional del Trabajo sobre la calidad del trabajo en la economía de plataformas ${ }^{64}$, la mayoría de las personas que trabajan en plataformas digitales alegan no existir suficientemente trabajo en la plataforma, lo que se traduce en que una parte importante del tiempo invertido en la plataforma se destina a esperar servicios o tareas o a buscar trabajo dentro del mercado interno de la plataforma ${ }^{65}$.

\footnotetext{
${ }^{60}$ Ver Mark ButLer, "Working time and flexibility in the United Kingdom", Comparative Labor Law Dossier. Working time and flexibility, IUSLabor no 1, 2016, p. 73-74.

${ }^{61}$ Ver Evangelos Angelopoulos y Panagiotis Boumpoucheropoulos, "Working time and flexibility in Greece", Comparative Labor Law Dossier. Working time and flexibility, IUSLabor no 1, 2016, p. 32-33.

${ }^{62}$ Ver Vincenzo Ferrante, "Working time and flexibility in Italy", Comparative Labor Law Dossier. Working time and flexibility, IUSLabor no 1, 2016, p. 42.

${ }^{63}$ Abi Adams, Mark Freedland y Jeremias Prassl, "The 'Zero-Hours Contract': Regulating Casual Work, or Legitimating Precarity?”, Oxford Legal Studies Research Paper, no 11, 2015, p. 19; Borja SuÁrez Corujo, "The Gig Economy and its Impact on Social Security: The Spanish example", European Journal of Social Security, vol. 19, no 4, p. 299; o Anna Ginès i Fabrellas, "The zero-hour contract in platform work...", op. cit., p. 10-11.

${ }^{64}$ Organización Internacional del Trabajo, "La calidad del trabajo en la economía de plataformas", Grupo 3: Tecnología al servicio del desarrollo social, ambiental y económico, Nota informativa no 5, 2018, p. 3.

${ }^{65}$ Janine BERG, Income security in the on-demand economy: findings and policy lessons from a survey of crowdworkers, Conditions of Work and Employment Series, no 74, Organización Internacional del Trabajo, Ginebra, 2016, p. 11-15 o Chris Forde et al., The Social Protection of Workers in the Platform Economy, op. cit., p. 45; Janine Berg, Marianne Furrer, Ellie Harmon, Uma Rani y M. Six Silberman, Digital labour platforms and the future of work. Towards decent work in the online world, Organización Internacional del Trabajo, Ginebra, 2018, p. 62.
} 
La contratación a demanda y la consideración como tiempo retribuido solamente el tiempo de prestación efectiva de servicios implica que no existe una correlación directa entre el tiempo de conexión a la plataforma y la retribución recibida por la persona. Ciertamente sí que existe una mínima correspondencia; según el número de horas de conexión a la plataforma, la persona trabajadora puede hacer una estimación de la retribución que espera obtener. Sin embargo, no existe una relación directa, pudiendo verse esta estimación afectada por las circunstancias concretas de la demanda o por la presencia de más personas trabajadoras también conectadas a la plataforma.

Este sistema de contratación y retribución afecta también a las decisiones de las personas trabajadoras respecto del número de horas de conexión a la plataforma. Las personas que trabajan en plataformas se ven obligadas a invertir más horas en la plataforma para alcanzar un número adecuado de tareas o servicios para obtener la retribución esperada.

Esta configuración del trabajo en plataformas genera un riesgo de (auto)explotación por parte de las personas trabajadoras, que ven como una parte del tiempo que se encuentran conectadas a la plataforma no es retribuido. Este riesgo es especialmente importante en el contexto del trabajo en plataformas, por cuanto la organización del trabajo se fundamenta en la sobre oferta de trabajo. Efectivamente, el crowdsourcing y la contratación a demanda requieren de un número elevado de personas conectadas a la plataforma para que puedan atender a la demanda que exista en todo, en cada y en cualquier momento en la plataforma. Es decir, la plataforma no tiene incentivos a limitar el número de personas conectadas en la plataforma para garantizar que la oferta sea la justa y necesaria para cubrir le demanda esperada. Todo lo contrario. El modelo de negocio de las plataformas digitales se fundamenta en la rápida conexión entre oferta y demanda de un servicio; se fundamenta en una sobre oferta de trabajo que permita esta casi inmediata conexión. En consecuencia, en muchas ocasiones existe un número de personas trabajadoras conectadas superior a las necesarias para atender la demanda esperada, lo que genera una fuerte competencia entre las personas trabajadoras para acceder a servicios o tareas. Con este modelo, las personas que trabajan en plataformas digitales se ven obligadas a invertir más horas y en horarios antisociales para acceder a un número adecuado de tareas o servicios para alcanzar la retribución esperada -en ocasiones, la retribución necesitada-.

Por todo lo anterior, el tiempo de trabajo en plataformas digitales es imprevisible. Como consecuencia de la utilización de facto del contrato de cero horas, que supone la contratación de las personas trabajadoras solamente cuando existen demandas de tareas o servicios, existe una imprevisibilidad e inseguridad en la determinación del tiempo de trabajo y retribución. La dependencia directa respecto de las demandas del mercado convierte el acceso a trabajo y remuneración en imprevisible, además de generar inestabilidad futura, al afectar a prestaciones públicas futuras ${ }^{66}$.

\subsection{Estrategias de gamificación e inseguridad algoritmica}

El tiempo de trabajo en el trabajo en plataformas digitales es, adicionalmente, imprevisible y, por tanto, inseguro, como consecuencia de la utilización de estrategias de gamificación y formas de dirección, organización y control algorítmico por parte de la plataforma. Como se ha apuntado anteriormente, la organización del trabajo en plataformas se determina mediante el uso de algoritmos, que distribuyen servicios y tareas en atención a métricas determinadas por la plataforma. Variables como el número de horas de conexión, conexión en días y horas de alta demanda, número de servi-

\footnotetext{
${ }^{6}$ Anna Ginès I FABrellas, "La desprotección social de los trabajadores en plataformas digitales", en Miguel RodriguEZ-PiÑERO Royo, Adrián Todolí Signes y Macarena Hernández Bejarano, Cambiando la forma de trabajar y de vivir: de las plataformas a la economía colaborativa real, Tirant lo Blanch, Valencia, 2020, p. 215-248.
} 
cios aceptados, servicios rechazados, etc. son utilizadas para determinar la asignación de servicios y tareas. Las personas con mejores métricas tienen acceso a más y mejores servicios y tareas.

No obstante, estas estrategias y fórmulas de organización y distribución de tareas en el mercado interno de la plataforma configuran el tiempo de trabajo en variable, inestable y, en esencia, inseguro. Por un lado, contribuyen a modular las decisiones de las personas trabajadoras para encajar con los intereses organizativos de la plataforma y no necesariamente con sus intereses personales y, por otro lado, contribuyen a generar todavía más inseguridad en el acceso a trabajo y remuneración.

Las estrategias de gamificación ${ }^{67}$ que utilizan las plataformas digitales inducen a las personas trabajadoras a conectarse a la plataforma, aceptar tareas o no rechazar servicios en contra de sus intereses. Efectivamente, las plataformas son conocidas por la utilización de estrategias de gamificación en la dirección y organización del trabajo, atribuyendo puntuaciones y valoraciones a las personas trabajadoras y estableciendo niveles o rankings para favorecer la competencia dentro del mercado interno de la plataforma y conseguir, así, modular el comportamiento de las personas trabajadoras según los intereses de la plataforma.

Las estrategias de gamificación son ciertamente efectivas. Flirteando con la adicción que crean, garantizan rapidez, efectividad y eficiencia en la ejecución del trabajo, además de motivar e involucrar a las personas trabajadoras con la empresa ${ }^{68}$. Sin embargo, también suponen una monitorización constante e intensificación del trabajo, que pueden traducirse en estrés, frustración e inseguridad ${ }^{69}$.

El modelo de organización del trabajo en las plataformas digitales requiere a las personas trabajadoras invertir una cuantía de tiempo considerable para poder alcanzar y mantener una posición aceptable dentro de la plataforma. Efectivamente, las personas que recién acceden a la plataforma tienen que invertir tiempo creando y construyendo una reputación personal, conectándose largas horas, aceptando todos los pedidos y no rechazando ninguno, para, como si de un videojuego se tratara, ir superando pantallas para alcanzar los niveles superiores. Incluso una vez dentro del sistema, las personas trabajadoras deben mantener una atención constante y milimétrica al trabajo para evitar afectar a su reputación. Una semana con menor actividad laboral -por causas, incluso, justificadas como responsabilidades familiares, laborales o una lesión- o una valoración mediocre por parte de una persona clienta -incluso, cuando injustificada o por causa no imputable a la persona trabajadora- pueden afectar significativamente la puntuación individual de la persona, afectando enormemente a su capacidad para generar ingresos la semana siguiente.

Más allá de estas estrategias de gamificación, la dirección y organización algorítmica provoca inseguridad en la determinación del tiempo de trabajo y retribución, por no existir transparencia en las reglas del juego. Las personas que trabajan en plataformas no conocen las métricas o variables utilizadas por la plataforma para determinar la distribución de servicios o tareas, asignación de franjas horarias o, incluso, desconexión de la plataforma ${ }^{70}$. La experiencia en la plataforma o la colaboración entre las personas trabajadoras ${ }^{71}$ puede generar una ligera intuición de las métricas utilizadas por la plataforma o, incluso, su ponderación. Sin embargo, no existe un conocimiento cierto de dichas métricas, lo que genera una nueva forma de inseguridad, denominada inseguridad

\footnotetext{
${ }^{67}$ Véase Sarah Mason, "High score, low pay: why the gig economy loves gamification”, The Guardian, 20.11 .2018 (disponible en: https://www.theguardian.com/business/2018/nov/20/high-score-low-pay-gamification-lyft-uber-drivers-ride-hailing-gig-economy?CMP=twt_gu; última consulta: 13.12.2020).

${ }^{68}$ Sarah Mason, "High score, low pay: why the gig economy loves gamification", op. cit.

${ }^{69}$ Leslie Ноок, "Uber: The uncomfortable view from the driving seat", Financial Times, 4.10.2017 (disponible en: https://www. ft.com/content/c9a8b592-a81d-11e7-ab55-27219df83c97; última consulta: 13.12.2020).

${ }^{70}$ Sangeet Paul Choudary, “The architecture of digital labour platforms...”, op. cit., p. 10 o Alex Rosenblat, Uberland. How algorithms are rewriting the rules of work, University of California Press, Oakland (Estados Unidos), 2018, p. 93.

${ }^{71}$ Este es el caso, por ejemplo, de Turkopticon, espacio de encuentro entre las personas que trabajan en Amazon Mechanical Turk para valorar a empresas clientas o compartir estrategias para mejorar la obtención de ingresos en la plataforma.
} 
algorítmica $^{72}$. Efectivamente, el tiempo de trabajo de las personas trabajadoras es determinado por sofisticados algoritmos que utilizan métricas por ellas desconocidas. El tiempo de trabajo y, por tanto, también la retribución de las personas que trabajan en plataformas depende de variables que, si bien basadas en su propia actuación en la plataforma, desconocen.

Más allá, es práctica habitual de las plataformas digitales modificar dichas métricas o variables sin informar previamente a las personas trabajadoras ${ }^{73}$. Las personas que trabajan en plataformas ven como los estándares utilizados por la plataforma para determinar la asignación de tareas o franjas horarios son modificados, sin información previa. La ausencia de información dificulta que puedan modificar su conducta para encajarla a las nuevas métricas de la plataforma, contribuyendo así a generar mayor inseguridad en el acceso a tareas y servicios y, en consecuencia, remuneración.

La opacidad en la configuración del algoritmo y la práctica de las empresas de modificar las métricas utilizadas para mejor adaptar la asignación de servicios y tareas a sus necesidades organizativas sin información a las personas trabajadoras genera una situación de especial inseguridad. Un modelo de organización del trabajo basado en la asignación de tareas y franjas horarias en atención a la actividad laboral pasada de las personas trabajadoras sin que estas conozcan las métricas utilizadas por la plataforma que, además, están sujetas a cambios constantes y desconocidos, generan una inseguridad extrema. Un modelo donde la distribución de trabajo en el mercado interno de la plataforma se realiza en función de las puntuaciones individuales de las personas trabajadoras, pero la fórmula de determinación de dichas puntuaciones es desconocida por las personas sujetas a las mismas, además de cruelmente desconsiderado, genera inseguridad. Una nueva forma de inseguridad, denominada inseguridad algorítmica, que obliga a las personas a conectarse a la plataforma, aceptar tareas y no rechazar servicios en contra de sus intereses individuales para evitar el riesgo de afectar a sus puntuaciones y, en consecuencia, a sus capacidades para obtener ingresos en el futuro ${ }^{74}$.

\section{La protección del trabajo en plataformas digitales}

\subsection{El trabajo en plataformas como trabajo por cuenta ajena}

El trabajo en plataformas digitales, sin perjuicio de los nuevos elementos de flexibilidad introducidos en la forma de organización del trabajo, es trabajo por cuenta ajena.

Las características del trabajo en plataformas digitales, como se ha analizado anteriormente, evidencian la existencia de ajenidad y dependencia propia de la relación laboral. El trabajo es prestado dentro del ámbito de dirección y organización de la plataforma, que gestiona los servicios y tareas según criterios organizativos propios para maximizar beneficios y asegurar la rentabilidad del negocio es un elemento claro de trabajo por cuenta ajena. Adicionalmente, la asunción de los costes y riesgos propiamente empresariales de diseño, creación y mantenimiento de la propia plataforma, la aplicación informática y el algoritmo, además de la intervención en el mercado mediante la adopción de todas las decisiones económicas, empresariales y estratégicas, evidencia la existencia de ajenidad.

En este sentido, la primera medida para avanzar en la protección del trabajo en plataformas es reconocer la legítima relación laboral. La protección de esta nueva forma de organización del trabajo requiere realizar una correcta valoración de la naturaleza jurídica que caracteriza la relación entre la

\footnotetext{
72 Alex Wood y Vili Lehdonvirta, "Platform Precarity: Surviving Algorithmic Insecurity in the Gig Economy", 31st Annual Meeting of the Society for the advancement of socio-economics. Fathomless Futures: Algorithmic and Imagined, Nueva York, 27-29.6.2019.

${ }^{73}$ Sangeet Paul CHOudary, "The architecture of digital labour platforms...", op. cit., p. 19.

${ }^{74}$ Jeremias PrassL, Humans as a service, op. cit., p. 55.
} 
plataforma y las personas trabajadores, sin caer en la tentación de pretender regular el fenómeno ex novo y ad hoc.

Un sector de la doctrina iuslaboralista se ha posicionado a favor de la creación de la figura del "trabajo independiente" como medida para proteger el trabajo en plataformas. Esta es la posición mantenida, por ejemplo, por Seth D. Harris y Alan B. Krueger ${ }^{75}$ o, en el ordenamiento jurídico español, por Jesús R. Mercader Uguina ${ }^{76}$ o Salvador del Rey Guanter ${ }^{77}$, que abogan por reformular la figura del TRADE para encajarla en el trabajo en plataformas y reconocerle derechos mínimos, tales como igualdad y no discriminación, prevención de riesgos laborales o negociación colectiva. En una línea similar, la asociación de empresas tecnológicas Adigital, entre las que se encuentran plataformas digitales como Uber, Glovo, Deliveroo o Stuart, también se posiciona a favor de la reformulación de la figura del TRADE para adaptarla al trabajo en plataformas ${ }^{78}$.

Otras personas de la doctrina iuslaboralista, defensoras de la existencia de relación laboral, defienden la necesidad de articular el trabajo en plataformas a través de una relación laboral de carácter especial. Este es el caso, por ejemplo, de Adrián Todolí Signes, que propone la regulación de una relación laboral especial para el trabajo en plataformas que reconozca libertad a las personas trabajadoras a determinar su horario y jornada ${ }^{79}$.

Desde mi punto de vista, no obstante, la protección del trabajo en plataformas digitales no requiere regulaciones $a d$ hoc ni especiales. Los cambios en la organización del trabajo introducidos por las plataformas digitales parecen que han venido para quedarse; el fenómeno del trabajo en plataformas digitales y las estrategias de dirección, organización y control algorítmico del trabajo parecen encontrarse todavía en una fase inicial con ánimo y capacidad para expandirse en el futuro y en otros ámbitos y sectores económicos. $Y$ es indudable que algunos de los cambios y novedades introducidas por esta nueva forma de organización del trabajo generan beneficios económicos, sociales e, incluso, laborales. Sin embargo, para que sean verdaderamente beneficiosos desde un punto de vista colectivo, es necesario que respeten las normas laborales existentes, sin que sus intereses particulares prevalgan en la determinación de la respuesta normativa.

En consecuencia, el trabajo en plataformas digitales debe regirse por la regulación laboral existente. Como defiende un sector importante de la doctrina iuslaboralista ${ }^{80}$, el trabajo en plataformas digitales debe calificarse como trabajo por cuenta ajena por concurrir las notas de dependencia y ajenidad propias de la relación laboral ${ }^{81}$. Efectivamente, como se ha apuntado, el trabajo en plataformas es trabajo desarrollado dentro del ámbito de dirección y organización de la plataforma, que organiza el trabajo en el mercado interno según criterios organizativos propios, y es trabajo por

\footnotetext{
${ }^{75}$ Véase, en este sentido, Seth D. Harris y Alan B. KRUEger, A proposal for modernizing Labor Laws for the twenty-first-century work: the "independent worker, The Hamilton Project, 2015.

${ }^{76}$ Jesús R. Mercader Uguina, "El nuevo modelo de trabajo autónomo en la prestación de servicios a través de plataformas digitales", Diario La Ley, no 9, 11.7.2017 y Jesús R. Mercader Uguina, "Los TRADE en las plataformas digitales", en Francisco Pérez de los Cobos (Director), El trabajo en plataformas digitales. Análisis sobre su situación jurídica y regulación futura, Wolters Kluwer, Las Rozas (Madrid), 2018 (versión digital).

${ }^{77}$ Salvador Del Rey Guanter (Director), Proyecto Technos. Economia de plataformas, Blockchain y su impacto en los Recursos Humanos y en el marco regulatorio de las relaciones laborales, Instituto Cuatrecasas, Wolters Kluwer, Las Rozas (Madrid), 2019, p. 280 y 286-289.

${ }^{78}$ Propuesta normativa en materia de trabajo en plataformas digitales, Asociación Española de la Economía Digital, julio 2019 (disponible en: https://www.adigital.org/media/propuesta-regulatoria-plataformas-digitales.pdf; última consulta: 13.12.2020).

${ }^{79}$ Adrián Todolí Signes, El trabajo en la era de la economía colaborativa, Tirant lo Blanch, Valencia, 2017, p. 71.

${ }^{80}$ Valerio de Stefano, "The rise of the «just-in-time workforce»...”, op. cit.; Jeremias Prassl, Humans as a service, op. cit.; Raquel Serrano Olivares, "Nuevas formas de organización empresarial: economía colaborativa -o mejor, economía digital a demanda-, trabajo 3.0 y laboralidad", en Miguel C. Rodríguez-Piñero Royo y Macarena Hernández Bejarano (Directores), Economía colaborativa y trabajo en plataforma: realidades y desafios, Editorial Bomarzo, Albacete, 2017, p. 19-49; Ignasi BeLtrán de Heredia Ruiz, Work in the platform economy: arguments for an employment relationship, Huygens Editorial, 2019, p. 113; o Borja SuÁrez CoRUJO, "The Gig Economy and its Impact on Social Security...", op. cit., p. 300, entre otras muchas personas.

${ }^{81}$ Ver también Anna Ginès I Fabrellas, El trabajo en plataformas digitales, op. cit.
} 
cuenta ajena por no gozar las personas trabajadoras de capacidad de emprendedoría para determinar los términos y condiciones de la actividad, ni asumir el riesgo y ventura de la misma.

Como se ha apuntado anteriormente, la última versión disponible de la propuesta de Real Decreto-ley sobre el trabajo en plataformas va en esta línea de reconocer la laboralidad del trabajo en plataformas. Así, se introduce una disposición adicional vigesimotercera en el Estatuto de los Trabajadores que establece que, por aplicación del artículo 8.1 ET, "se presume incluida en el ámbito de esta ley la actividad de las personas que presten servicios retribuidos consistentes en el reparto o distribución de cualquier producto de consumo o mercancia, por parte de empleadoras que ejercen las facultades empresariales de organización, dirección y control de forma directa, indirecta o implícita, mediante una gestión algoritmica del servicio o de las condiciones de trabajo, a través de una plataforma digital".

Es indudable el interés político, social y estratégico de regular el trabajo en plataformas para ofrecer una respuesta normativa definitiva al conflicto. Sin embargo, debe cuestionarse si esta propuesta de regulación alcanzará tal objetivo. La regulación parcial del fenómeno del trabajo en plataformas mantiene ampliamente abierto el debate de la naturaleza jurídica del trabajo en otras modalidades de plataformas, como las de trabajos de cuidado, limpieza o las inabarcables plataformas de (micro)tareas online.

Desde mi punto de vista, la respuesta normativa debe reforzar instituciones laborales estables, como la presunción de laboralidad del artículo 8 ET, y aprovechar la oportunidad para solventar el extendido y devastador problema del falso trabajo autónomo en el mercado de trabajo español.

\subsection{Propuestas en materia de tiempo de trabajo}

Más allá de la consideración del trabajo en plataformas digitales como trabajo por cuenta ajena y de descartar la necesidad de adaptar la regulación legal a un específico modelo de negocio, la protección del trabajo en plataformas va más allá. Efectivamente, como se ha concluido en el presente trabajo, el modelo de precariedad laboral que introducen las plataformas digitales va más allá de su consideración como falso trabajo autónomo, sino que viene determinado por características propias del modelo de negocio, tales como el crowdsourcing y la contratación a demanda.

En este sentido, a continuación, se incluyen cuatro propuestas en materia de tiempo de trabajo que, dentro de la actual regulación laboral, pretenden garantizar la protección del trabajo en plataformas.

En primer lugar, es necesario vehicular el trabajo en plataformas digitales a través del contrato a tiempo parcial, que ofrece un nivel de flexibilidad suficiente para adaptarse al modelo de negocio de las plataformas digitales, a la vez que ofrece una mínima seguridad y estabilidad jurídica a las personas trabajadoras.

Como es bien sabido, el trabajo a tiempo parcial permite la realización de horas complementarias por encima de las horas ordinarias pactadas, ofreciendo una flexibilidad considerable. Según el artículo 12.5 ET, las personas contratadas a tiempo parcial con una jornada superior a 10 horas semanales pueden suscribir un pacto de horas complementarias hasta el $30 \%$ de la jornada ordinaria pactada o, incluso, del 60\% si así está previsto en convenio colectivo. Más allá, las personas con una jornada superior a 10 horas semanales y contrato indefinido pueden realizar, adicionalmente, horas complementarias de aceptación voluntaria hasta el 15\% de las horas ordinarias pactadas o, incluso, el 30\% si así está previsto por convenio colectivo. Como puede observarse, por tanto, el contrato a tiempo parcial ofrece una flexibilidad importante en materia de tiempo de trabajo, que permite la realización de horas complementarias pactadas y de aceptación voluntaria hasta el 45\% de la jornada ordinaria pactada -0 , incluso, $90 \%$ si así está previsto en convenio colectivo-. 
Sin pretender defender la elevada -incluso, excesiva - flexibilidad que ofrece el contrato a tiempo parcial $^{82}$, resulta adecuado para vehicular el trabajo en plataformas. Por un lado, por cuanto resulta una modalidad contractual disponible en el actual ordenamiento jurídico-laboral, sin requerir una actuación normativa específica. Y, por otro lado, por cuando, sin renunciar a la flexibilidad característica del trabajo en plataformas en la gestión del tiempo de trabajo, ofrece una mínima seguridad y estabilidad a las personas trabajadoras requiriendo una jornada mínima de 10 horas para acceder a horas complementaras pactadas y, adicionalmente, una relación laboral indefinida para acceder a las de aceptación voluntaria.

En segundo lugar, todo el tiempo de prestación de servicios debe ser considerado tiempo de trabajo y, por tanto, tiempo retribuido. Es decir, tanto el tiempo de ejecución de servicios o tareas, como el tiempo de conexión a la plataforma, debe tener la consideración de tiempo de trabajo y, por tanto, tiempo retribuido ${ }^{83}$.

La exclusión del tiempo de conexión a la plataforma del tiempo de trabajo retribuido es contraria a la regulación comunitaria. Como es bien sabido, el derecho de la Unión Europea utiliza un concepto binario del tiempo de trabajo, según el cual es tiempo de trabajo "todo período durante el cual el trabajador permanezca en el trabajo, a disposición del empresario y en ejercicio de su actividad o de sus funciones, de conformidad con las legislaciones y/o prácticas nacionales" $"$, y es tiempo de descanso el tiempo restante ${ }^{85}$. Es decir, según el derecho comunitaria existe solamente tiempo de trabajo o tiempo de descanso, no reconociéndose una categoría intermedia de tiempo de disponibilidad. Por tanto, este tiempo que la persona trabajadora está disponible para la empresa -o, en el caso de la plataforma, el tiempo que está conectada a la plataforma y a la espera de recibir servicios- es tiempo de trabajo. Efectivamente, tiene la consideración de tiempo de trabajo por cuanto, siguiendo la anterior definición, la persona trabajadora se encuentra "a disposición del empresario y en ejercicio de su actividad"; la persona trabajadora se encuentra disponible para atender rápidamente a los servicios y pedidos que la empresa le asigne.

Es más, incluso en el caso de considerarse que durante dicho tiempo de conexión a la plataforma la persona no se encuentra "en ejercicio de su actividad", este tiempo de conexión tiene la consideración de tiempo de trabajo. Efectivamente, la consideración como tiempo de trabajo del tiempo de conexión a la plataforma encaja con la doctrina del Tribunal de Justicia de la Unión Europea referente a las guardias localizadas. Según el tribunal, cuando la persona trabajadora está localizada, aun cuando fuera de las instalaciones de la empresa, y debe responder de inmediato o de forma rápida a la petición de la empresa, este tiempo tiene la consideración de tiempo de trabajo ${ }^{86}$. Por tanto, el tiempo de conexión a la plataforma debe ser considerado, a la luz del derecho de la Unión Europea, como tiempo de trabajo, sin perjuicio de la posibilidad de la plataforma de retribuir de manera diferente el tiempo de conexión y el tiempo de prestación efectiva de servicios ${ }^{87}$.

En tercer lugar, es esencial que la configuración del tiempo de trabajo en plataformas digitales respete las exigencias de transparencia y previsibilidad de la Directiva 2019/1152 del Parlamento Europeo y del Consejo, de 20 de junio de 2019, relativa a unas condiciones laborales transparentes

\footnotetext{
${ }^{82}$ Anna Ginès i Fabrellas, “Contrato para la formación y el aprendizaje y contrato a tiempo parcial”, en Salvador DEL Rey GuANTER (Director), Las novedades de la Ley 3/2012, de reforma del mercado laboral, y su impacto en el sistema de relaciones laborales, La Ley, Madrid, 2013, p. 105.

${ }^{83}$ Organización Internacional del Trabajo, Work for a brighter future. Global Commission on the Future of Work, Ginebra, 2019 , p. 41.

${ }^{84}$ Artículo 2.1 de la Directiva 2003/88/CE del Parlamento Europeo y del Consejo, de 4 de noviembre de 2003, relativa a determinados aspectos de la ordenación del tiempo de trabajo.

${ }^{85}$ Ver, por todas, STJUE de 3.10.2000, asunto Simap (C-303/98).

${ }^{86}$ STJUE de 21.2.2018, asunto Matzak (C-518/15).

${ }^{87}$ STJUE de 10.9.2015, asunto Tyko (C-266/14).
} 
y previsibles en la Unión Europea que, según su artículo 21, deben introducirse por parte de los estados miembros antes del 1 de agosto de 2022.

La Directiva comunitaria, con la finalidad de mejorar las condiciones laborales mediante la transparencia y la previsibilidad, obliga a la empresa a proporcionar a las personas trabajadoras información escrita sobre las condiciones laborales básicas - tales como denominación de las partes, lugar de trabajo, fecha de inicio y, en su caso, de finalización, duración del contrato, retribución o tiempo de trabajo- al inicio de la relación laboral (artículos 4 y 5).

Adicionalmente, contiene cuatro previsiones especialmente importantes para el trabajo en plataformas digitales. La Directiva establece disposiciones concretas para garantizar una mínima seguridad y previsibilidad en las condiciones laborales en aquellos trabajos donde el tiempo de trabajo es total o mayoritariamente imprevisible, como sucede mayoritariamente en el trabajo en plataformas.

Primero, establece una obligación de información hacia las personas trabajadoras. El artículo 4.2.m) de la Directiva exige a la empresa a informar a las personas trabajadoras acerca de (i) la variabilidad de horarios, (ii) el número de horas retribuidas garantizadas, en su caso, (iii) la retribución por horas adicionales, (iv) los días o franjas horarias en las que las personas pueden ser llamadas para prestar servicios o (v) la antelación mínima con la que la empresa debe informar antes de iniciar el trabajo. En cuanto al preaviso mínimo para la realización de tareas o servicios, es importante destacar que el artículo 12.5 ET exige un preaviso de 3 días en la realización de horas complementarias en el contrato a tiempo parcial. Si bien este preaviso podría identificarse como incompatible con la dinámica propia y característica del trabajo en plataformas, nótese que por convenio colectivo podría fijarse un preaviso inferior.

Segundo, el artículo 10 de la Directiva establece una obligación de mínima previsibilidad del trabajo. Así, su apartado primero obliga a los estados miembros a adoptar medidas para garantizar que la petición de trabajo o la asignación de tareas por parte de la empresa a las personas trabajadoras respete el preaviso fijado legal o convencionalmente y el trabajo tenga lugar en los días o franjas horarias determinadas con antelación por la empresa. En caso contrario, el apartado segundo de dicho precepto reconoce el derecho de la persona trabajadora a rechazar la tarea asignada sin sufrir ninguna consecuencia desfavorable.

Tercero, el apartado tercero de este mismo precepto establece una previsión para ofrecer mínima seguridad a las personas cuyo trabajo es total o mayoritariamente imprevisible. Así, los estados miembros deben prever medidas para que las personas trabajadoras reciban una indemnización en caso de cancelación de tareas o servicios sin observar un plazo de preaviso determinado. Es decir, las personas trabajadoras tienen derecho a percibir una indemnización por cancelaciones in extremis de tareas o servicios.

Cuarto, el artículo 11 de la Directiva establece disposiciones concretas para los estados que admiten contratos de cero horas sin una jornada mínima garantizada. Si bien este no es el caso del ordenamiento jurídico-laboral espańol, es interesante apuntar que la Directiva obliga a los estados miembros a adoptar medidas para evitar prácticas abusivas, tales como una presunción de laboralidad de las personas que trabajan un mínimo de horas o limitaciones al uso o duración de dichos contratos.

Finalmente, en cuarto lugar, como medida para eliminar o reducir la inseguridad algorítmica propia del trabajo en plataformas digitales, es importante reconocer el derecho de las personas trabajadoras a conocer la lógica aplicada por la empresa en la distribución de tareas, asignación de franjas horarias, retribución o desconexión de la plataforma ${ }^{88}$.

\footnotetext{
${ }^{88}$ En este sentido, el informe de la Organización Internacional del Trabajo de 2021 sobre trabajo en plataformas incluye la transparencia y responsabilidad de los algoritmos por parte de empresas y personas trabajadoras como medida para avanzar en la protección del trabajo en plataformas digitales (ver Organización Internacional del Trabajo, World Employment and Social Outlook. The role of digital labour platforms in transforming the world of work, Organización Internacional del Trabajo, 2021, p. 256).
} 
El derecho o no de las persones trabajadoras de conocer el algoritmo utilizado por las plataformas digitales pareció ser uno de los puntos de desacuerdo más importantes entre agentes sociales en la mesa social para la regulación del trabajo en plataformas y se ha excluido de la propuesta final de regulación de la denominada "Ley Rider" ${ }^{29}$. Efectivamente, mientras que los sindicatos abogaban por reconocer este derecho a las personas trabajadoras, la patronal se escudaba detrás de la consideración del algoritmo como secreto profesional.

La última versión del Real Decreto-ley referente al trabajo en plataformas, no obstante, introduce un derecho de información del algoritmo a la representación legal de la plantilla. En concreto, introduce un nuevo apartado al artículo 64.4 ET según el cual la representación legal de la plantilla tendrá derecho a ser informada por la empresa "de los parámetros, reglas e instrucciones en los que se basan los algoritmos o sistemas de inteligencia artificial que afecten a la toma de decisiones que pueden incidir en las condiciones de trabajo, el acceso y mantenimiento del empleo, incluida la elaboración de perfiles". Se reconoce, por tanto, un nuevo derecho de información de la representación legal de la plantilla de las métricas del algoritmo utilizado por la empresa para la toma de decisiones automatizadas y la elaboración de perfiles en el ámbito laboral. Es importante destacar que este derecho de información se reconoce respecto de todas las empresas que utilicen algoritmos o sistemas de inteligencia artificial y no solamente respecto de las plataformas digitales.

En todo caso, sin perjuicio de la valoración positiva que merece este nuevo derecho de información de la representación legal de la plantilla, es criticable que no se aproveche la oportunidad para dotar de contenido un derecho ya existente como es el derecho de las personas trabajadoras de conocer la lógica del algoritmo.

Sin perjuicio de aceptar la consideración del algoritmo como secreto profesional, lo cierto es que el derecho de las persones trabajadoras de conocer la lógica utilizada en la dirección y organización algorítmica ya se encuentra legalmente reconocido. Efectivamente, el artículo 14.2.g) del Reglamento General de Protección de Datos ${ }^{90}$, en relación con su artículo 22, establece que las personas trabajadoras sujetas a decisiones automatizadas con efectos jurídicos o significativamente similares tienen derecho a conocer la existencia de decisiones automatizadas, información significativa sobre la lógica aplicada, la importancia y las consecuencias que pueden derivarse de las decisiones automatizadas. Es decir, aquellas personas que están sujetas a decisiones automatizadas sin intervención humana alguna con efectos jurídicos o significativamente similares -tales como, por ejemplo, acceso a empleo o retribución-, tienen derecho a conocer la lógica aplicada en dicha decisión automatizada. En concreto, tienen derecho a conocer (i) la utilización por parte de la empresa de fórmulas de dirección y organización del trabajo automatizadas sin intervención humana, (ii) la lógica utilizada para la toma de dichas decisiones y (iii) la importancia y consecuencias que pueden derivarse de dichas decisiones automatizadas.

El derecho a obtener información sobre la lógica utilizada para la toma de decisiones automatizadas no puede identificarse con el derecho a conocer el algoritmo en sí mismo; no solo por constituir, efectivamente, un secreto empresarial que determina la menor o mayor rentabilidad del negocio, sino por resultar absolutamente contraproducente, por cuanto el algoritmo -o algoritmos, al utilizar en muchas ocasiones múltiples algoritmos- puede consistir en páginas y páginas de códigos indescifrables. Según establece el Grupo de Trabajo del Artículo 29, el derecho de

\footnotetext{
${ }^{89}$ Paula Solanas Alfaro y Núria Rius Montaner, "La "Ley rider" se encalla y renuncia a revelar los algoritmes de las apps", ara.cat, 7.12.2020 (disponible en: https://www.ara.cat/es/rider-encalla-renuncia-revelar-algoritmos_0_2577342320.html; última consulta: 13.12 .2020 ).

${ }^{90}$ Reglamento (EU) 2016/679 del Parlamento Europeo y del Consejo de 27 de abril de 2016 relativo a la protección de las personas físicas en lo que respecta al tratamiento de datos personales y a la libre circulación de estos datos y por el que se deroga la Directiva 95/46/CE.
} 
información del artículo 14.2.g) del Reglamento General de Protección de Datos, basado en el principio fundamental de transparencia en materia de protección de datos, se refiere al derecho a obtener información clara y simple sobre el funcionamiento del proceso de adopción de decisiones de forma automatizada ${ }^{91}$.

En el marco de las plataformas digitales, desde mi punto de vista, este derecho de información implica el derecho a obtener información sobre las variables o métricas utilizadas por el algoritmo para la distribución de tareas, asignación de franjas horarias, retribución o desconexión de la plataforma; conocer también su ponderación o importancia relativa dentro de la ecuación; y, finalmente, conocer las consecuencias que pueden derivarse en caso de no alcanzar dichas métricas o estándares.

\section{Conclusiones}

A modo de conclusión, el trabajo en plataformas digitales es trabajo propio de la relación laboral. Es más, teniendo en cuenta las características intrínsecas del trabajo a demanda y su impacto sobre el tiempo de trabajo y remuneración, la relación laboral es esencial para garantizar mínima estabilidad y dignidad en el trabajo en plataformas.

La actual definición de trabajo por cuenta ajena y el régimen jurídico del contrato a tiempo parcial ofrecen la suficiente flexibilidad para encajar el modelo de negocio de las plataformas digitales en la regulación laboral actual, sin que resulten necesarias soluciones normativas artificiales para adoptar la regulación laboral a un modelo basado, precisamente, en eludir dicha norma laboral.

Asimismo, el trabajo en plataformas debe respetar la norma comunitaria en materia de tiempo de trabajo y protección de datos, debiéndose considerar como tiempo de trabajo retribuido el tiempo de conexión a la plataforma y reconociéndose el derecho de las personas trabajadoras a obtener información sobre la lógica aplicada por la plataforma en la dirección y organización algorítmica del trabajo.

Las plataformas digitales han venido para quedarse y los beneficios empresariales y sociales que generan no pueden ignorarse, pero para que generen beneficios para la sociedad en general y no solamente beneficios privados y particulares, es necesario, urgente e inevitable que respeten la normativa laboral, exigible, por otro lado, a toda empresa, negocio o actividad.

\section{Bibliografía}

Abi Adams, Mark Freedland y Jeremias Prassl, "The 'Zero-Hours Contract': Regulating Casual Work, or Legitimating Precarity?”, Oxford Legal Studies Research Paper, no 11, 2015, p. 1-21.

Article 29 Data Protection Working Party, Guidelines on Automated individual decision-making and Profiling for the purposes of Regulation 2016/679, 3.10.2017.

RubÉn Agote, "On demand economy: 10 claves de entendimiento laboral", IUSLabor, no 1, 20I7, p. 1-18.

Henar Álunarez Cuesta, El futuro del trabajo vs. el trabajo del futuro. Implicaciones laborales de la industria 4.0, Colex, A Coruña, 2017.

Evangelos Angelopoulos y Panagiotis Boumpoucheropoulos, "Working time and flexibility in Greece", Comparative Labor Law Dossier. Working time and flexibility, IUSLabor n ${ }^{\circ} 1$, 2016, p. 23-35.

\footnotetext{
${ }^{91}$ Article 29 Data Protection Working Party, Guidelines on Automated individual decision-making and Profiling for the purposes of Regulation 2016/679, 3.10.2017, p. 16-17.
} 
Philippe Auvergnon, "Angustias de uberización y retos que plantea el trabajo digital al Derecho Laboral", Revista Derecho Social y Empresa (número especial: Nuevas formas de trabajo. Retos presentes y futuros), no 6, 2016, p. 25-42.

Ignasi Beltrán de Heredia Ruiz, "Employment status of platform workers (national courts decisions overview - Australia, Brazil, Chile, France, Italy, United Kingdom, United States \& Spain), Blog Una mirada critica a las relaciones laborales, (disponible en: https://ignasibeltran. com/2018/12/09/employment-status-of-platform-workers-national-courts-decisions-overview-australia-brazil-chile-france-italy-united-kingdom-united-states-spain).

Janine Berg, Marianne Furrer, Ellie Harmon, Uma Rani y M. Six Silberman, Digital labour platforms and the future of work. Towards decent work in the online world, Organizacion Internacional del Trabajo, Ginebra, 2018.

JANINE BERG, Income security in the on-demand economy: findings and policy lessons from a survey of crowdworkers, Conditions of Work and Employment Series, no 74, Organización Internacional del Trabajo, Ginebra, 2016.

Birgitta Bergvall-Kåreborn y Debra Howcroft, "Amazon Mechanical Turk and the commodification of labor", New Technology, Work and Employment, vol. 29, no 3, 2014, p. 213-223.

Alan Bogg, "Escaping Labour Law's Matrix: a review essay", Law Research Paper Series, no 5, University of Bristol, 2019, p. 1-11.

Daren C. Bradham, Crowdsourcing, MIT Press, Cambridge, 2013.

Mark Butler, "Working time and flexibility in the United Kingdom", Comparative Labor Law Dossier. Working time and flexibility, IUSLabor no 1, 2016, p. 66-74.

Miriam A.Cherry, "Beyond misclassification: the digital transformation of work", Comparative Labor Law \& Policy Journal, vol. 37, no 3, 2016, p. 577-602.

Sangeet Paul Choudary, "The architecture of digital labour platforms: policy recommendations on platform design for worker well-being”, ILO Future of Work Research Paper Series, no 3, 2018, p. 1-49.

Emanuele Dagnino, "Uber law: perspectiva jurídico-laboral de la sharing / on-demand economy", Revista Internacional y Comparada de Relaciones Laborales y Derecho del Empleo, vol. 3, n. 3, 2015, p. 1-31.

Valerio de Stefano y Antonio Aloisi, "Fundamental labour rights, platform work and humanrights protection of non-standard workers", en Janice R. Bellace y Beryl ter HaAR (Editores), Research Handbook on Labour, Business and Human Rights Law, Edward Elgar, Reino Unido, 2019, p. 359-379.

Valerio de Stefano, "The rise of the «just-in-time workforce»: On-demand work, crowdwork and labour protection in the "gig-economy", Conditions of Work and Employment Series, no 71, Organización Internacional del Trabajo, 2016

Salvador del Rey Guanter (Director), Proyecto Technos. Economia de plataformas, Blockchain y su impacto en los Recursos Humanos y en el marco regulatorio de las relaciones laborales, Instituto Cuatrecasas, Wolters Kluwer, Las Rozas (Madrid), 2019.

Vincenzo Ferrante, "Working time and flexibility in Italy", Comparative Labor Law Dossier. Working time and flexibility, IUSLabor no 1, 2016, p. 36-43.

Chris Forde, Mark Stuart, Simon Joyce, Liz Oliver, Danat Valizade, Gabriella Alberti, Kate Hardy, Vera Trappmann, Charles Umney y Calum Carson, The Social Protection of Workers in the Platform Economy, Directorate General for Internal Policies, Policy Department A: Economic and Scientific Policy, Parlamento Europeo, noviembre 2017.

AnNa Ginès i Fabrellas, "Contrato para la formación y el aprendizaje y contrato a tiempo parcial”, en Salvador Del Rey GuANTER (Director), Las novedades de la Ley 3/2012, de reforma 
del mercado laboral, y su impacto en el sistema de relaciones laborales, La Ley, Madrid, 2013, p. 63-122.

AnNa Ginès i Fabrellas, "La desprotección social de los trabajadores en plataformas digitales", en Miguel Rodriguez-Piñero Royo, Adrián Todolí Signes y Macarena Hernández BejaraNo, Cambiando la forma de trabajar y de vivir: de las plataformas a la economía colaborativa real, Tirant lo Blanch, Valencia, 2020, p. 215-248.

AnNa Ginès i Fabrellas, "The zero-hour contract in platform work. Should we ban it or embrace it?”, IDP. Revista de Internet, Derecho y Politica, no 28, 2019, p. 1-15.

Anna Ginès i Fabrellas, El trabajo en plataformas digitales. Nuevas formas de precariedad laboral, Thomson Reuters Aranzadi, Cizur Menor (Navarra), 2021.

Seth D. Harris y Alan B. Krueger, A proposal for modernizing Labor Laws for the twenty-firstcentury work: the "independent worker, The Hamilton Project, 2015.

Manuel Alejandro Hidalgo, El empleo del futuro. Un análisis del impacto de las nuevas tecnologías en el mercado laboral, Deusto, Barcelona, 2018.

Ursula Huws, Neil H. Spencer, Dag S. Syrdal y Kaire Holts, Work in the European Gig Economy. Research results from the UK, Sweden, Germany, Austria, The Netherlands, Switzerland and Italy, Foundation for European Progressive Studies, UNI Europa y University of Hertfordshire, 2017.

Sarah Kessler, Gigged. The Gig Economy, the end of the job and the future of work, Business Books, Londres, 2018.

Min Kyung Lee, Daniel Kusbit, Evan Metsky y Laura Dabbish, "Working with machines: the impact of algorithmic and data-driven management on human workers", Conference CHI'15 Proceedings of the 33rd Annual ACM Conference on Human Factors in Computing Systems, 2015, p. 1-10.

Jesús R. Mercader Uguina, "El nuevo modelo de Trabajo Autónomo en la prestación de servicios a través de plataformas digitales", Diario La Ley, no 9, 11.7.2017.

Jesús R. Mercader Uguina, "Los TRADE en las plataformas digitales", en Francisco Pérez de Lоs Совоs (Director), El trabajo en plataformas digitales. Análisis sobre su situación jurídica y regulación futura, Wolters Kluwer, Las Rozas (Madrid), 2018 (versión digital).

Jesús R. Mercader Uguina, El futuro del trabajo en la era de la digitalización y la robótica, Tirant lo Blanch, Valencia, 2017.

Organización Internacional del Trabajo, "La calidad del trabajo en la economía de plataformas”, Grupo 3: Tecnología al servicio del desarrollo social, ambiental y económico, Nota informativa no $5,2018$.

Organización InTERnacional del Trabajo, Work for a brighter future. Global Commission on the Future of Work, Ginebra, 2019.

Organización Internacional del Trabajo, World Employment and Social Outlook. The role of digital labour platforms in transforming the world of work, Organización Internacional del Trabajo, 2021.

Annarosa Pesole, Maria Cesira Urzí Brancati, Enrique Fernández-Macías, Federico Biagi e Ignacio González Vázquez, Platform Workers in Europe, JRC Science for Policy Report, Publications Office of the European Union, Luxemburgo, 2018.

Jeremias Prassl y Martin Risak, "Uber, TaskRabbit, \& Co: Platforms as Employers? Rethinking the Legal Analysis of Crowdwork", Comparative Labor Law Policy Journal, vol. 37, $\mathrm{n}^{\circ} 3$, 2016, p. 619-652.

Jeremias Prassl, Humans as a service. The promise and perils of work in the gig economy, Oxford University Press, Nueva York, 2018. 
Miguel Rodríguez-Piñero Royo, "La figura del trabajador de plataforma: las relaciones entre las plataformas digitales y los trabajadores que prestan sus servicios", en Francisco PÉREZ DE LOS Совоs (Director), El trabajo en plataformas digitales. Análisis sobre su situación jurídica y regulación futura, Wolters Kluwer, Las Rozas (Madrid), 2018 (versión digital).

Brishen Rogers, "The Social Costs of Uber", The University of Chicago Law Review Dialogue, vol. 82, 2015, p. 85-102.

Alex Rosenblat y Luke Stark, "Algorithmic labor and information asymmetries: a case study of Uber's drivers”, International Journal of Communication, vol. 10, 2016, p. 3758-3784.

Alex Rosenblat, Uberland. How algorithms are rewriting the rules of work, University of California Press, Oakland (Estados Unidos), 2018.

Juana María Serrano García, "La "uberización” del trabajo: una forma más de violencia laboral”, en Lourdes Mella MÉndez (Directora), Los actuales cambios sociales y laborales: nuevos retos para el mundo del trabajo, vol. 1, Peter Lang, Bern, 2017, p. 209-228.

Raquel Serrano Olivares, "Nuevas formas de organización empresarial: economía colaborativa -o mejor, economía digital a demanda-, trabajo 3.0 y laboralidad", en Miguel C. Rodríguez-Piñero Royo y Macarena Hernández Bejarano (Directores), Economía colaborativa y trabajo en plataforma: realidades y desafíos, Editorial Bomarzo, Albacete, 2017, p. 19-49.

Borja SuÁrez Corujo, "The 'Gig' Economy and its Impact on Social Security: The Spanish example”, European Journal of Social Security, vol. 19, no 4, p. 293-312.

Adrián Todolí Signes, "Nuevos indicios de laboralidad como resultado de las nuevas empresas digitales”, en Miguel C. Rodríguez-Piñero Royo y Macarena Hernández Bejarano (Directores), Economía colaborativa y trabajo en plataforma: realidades y desafios, Editorial Bomarzo, Albacete, 2017, p. 223-241.

Adrián Todolí Signes, El trabajo en la era de la economía colaborativa, Tirant lo Blanch, Valencia, 2017.

Maria Cesira Urzí Brancati, Annarosa Pesole y Enrique Fernández-Macías, New evidence on platform workers in Europe. Results from the second COLLEEM survey, JRC Science for Policy Report, Publications Office of the European Union, Luxemburgo, 2020.

Alex Wood y Vili Lehdonvirta, "Platform Precarity: Surviving Algorithmic Insecurity in the Gig Economy", paper congreso Fathomless Futures: Algorithmic and Imagined, Society for the advancement of socio-economics, Nueva York, 27-29.6.2019. 